\title{
Permanent Pacemaker Implantation After Transcatheter Aortic Valve Replacement: A Cost Analysis
}

Mansoor Ahmad ${ }^{1}$, Jay N. Patel ${ }^{2}$, Brian L. Loc ${ }^{2}$, Sharath C. Vipparthy ${ }^{2}$, Chirag Divecha ${ }^{2}$, Pablo X. Barzallo $^{2}$, Minchul Kim ${ }^{1}$, Timir Baman ${ }^{2}$, Marco Barzallo ${ }^{2}$, Sudhir Mungee ${ }^{2}$

1. Internal Medicine, University of Illinois College of Medicine at Peoria, Peoria, USA 2. Cardiology, University of Illinois College of Medicine at Peoria, Peoria, USA

Corresponding author: Mansoor Ahmad,mahmad1@uic.edu

\section{Abstract}

\section{Background}

Transcatheter aortic valve replacement (TAVR) can be complicated with a complete atrioventricular block requiring permanent pacemaker (PPM) implantation. The cost of index hospitalization for such patients is higher than usual. However, the magnitude of this increased cost is uncertain. We have looked at our fiveyear TAVR experience to analyze the detailed cost for PPM implantation in TAVR.

\section{Methods}

This study is a retrospective analysis of patients undergoing TAVR at our tertiary care center from December 2012 to April 2018. The initial sample size was 449. We excluded patients with prior PPM or an implantable cardioverter defibrillator (37). Patients who had their procedure aborted or required a cardiopulmonary bypass (16) and those with missing data variables (14) were excluded. The final sample size was 382 . The cost for admission was calculated as the US dollars incurred by the hospital. Cohort costs were categorized as a direct cost, which is patient based, and an indirect cost, which represents overhead costs and is independent of patient volume. Patients were divided into two groups based on the placement of PPM after TAVR. Chi-square test, t-test, and logistic linear regression were used for the statistical analysis.

\section{Results}

Of 382 patients, 19 (4.9\%) required PPM after TAVR. Baseline variables, including age, gender, and BMI, were not statistically significant. The PPM group had a significantly longer intensive care unit (ICU) stay (48.6 hours vs. 36.7 hours; $\mathrm{p}<0.001$ ) and total stay in the hospital (4.2 days vs. 3.4 days; $\mathrm{p}=0.047$ ). PPM implantation after TAVR increased cost on an average of $\$ 10,213$ more than a typical TAVR admission $(\mathrm{p}=0.04)$. The direct cost was also significantly high for the PPM group $(\$ 7,087 ; \mathrm{p}=0.02)$. On detailed analysis, almost all major cost categories showed a higher cost for pacemaker patients when compared with control.

Received 06/20/2019

Review began 06/24/2019 Review ended 06/25/2019 Published 06/26/2019

(c) Copyright 2019

Ahmad et al. This is an open access article distributed under the terms of the Creative Commons Attribution License CC-BY 3.0., which permits unrestricted use, distribution, and reproduction in any medium, provided the original author and source are credited.

\section{Conclusions}

PPM implantation adds a significant cost burden to TAVR admissions.

\section{Categories: Cardiology}

Keywords: permanent pacemaker, transcatheter aortic valve replacement, cost

\section{Introduction}

Aortic stenosis is an insidious disease with a long latency period, followed by rapid progression after the appearance of symptoms, resulting in a high rate of death (approximately $50 \%$ in the first two years after symptoms appear) among untreated patients. Surgical aortic valve replacement (SAVR) reduces symptoms and improves survival in patients with aortic stenosis [1-3] and has historically been the standard of care. The first transcatheter aortic valve replacement (TAVR) was performed in 2002, and TAVR has, since then, grown in popularity [4-6].

When compared with SAVR, TAVR has demonstrated improved three-year clinical outcomes, including allcause mortality, in the incidence of stroke and aortic valve hemodynamics [7]. However, these benefits can be offset by a much higher permanent pacemaker (PPM) implantation rate of 5\%-25\%, as SAVR has a PPM implantation rate of $1 \%$ per year [8]. The mechanism of atrioventricular (AV) block in TAVR is likely the direct injury to the His bundle, given its proximity to the membranous septum and native aortic valve [9-11]. Preexisting right bundle branch block (RBBB), low implantation, and the use of self-expanding valves (SEV) have been identified as common and consistent risk factors for PPM implantation across multiple studies [12-18]; a post-TAVR PPM implantation range from 6.0\% for the Edwards SAPIEN (Edwards Lifesciences, 
Irvine, CA, US) balloon-expandable valve (BEV) to $25 \%$ for the Medtronic CoreValve (Medtronic, Inc., Minneapolis, MN, US), a self-expanding valve [19-22].

There is a paucity of data on the cost impact of PPM implantation after TAVR. The majority of data show a higher cost with PPM implantation post-TAVR [12]. However, studies have shown no significant cost difference after PPM implantation in the TAVR population [23]. One recent study has shown no significant difference in cost at one year after TAVR [23]. Review of the literature demonstrated a gap in knowledge regarding cost breakdown for this population. In this study, we have attempted to separate the cost into direct, indirect, and total costs for TAVR admission. In addition, we acquired the charged incurred by each department involved in patient care for TAVR admission, to elaborate on the areas that are impacted by PPM implantation in the TAVR population.

In addition to cost, we have looked at other outcomes, including the intensive care unit (ICU) stay, the total length of stay (LOS) in hospital, and 30-day readmission and mortality.

The abstract for this article was presented at the Society of Cardiovascular Angiography and Intervention Conference 2019 as a poster [24].

\section{Materials And Methods \\ Patient population and study design}

This is a retrospective chart review of 449 patients who received TAVR at OSF Saint Francis Medical Center between December 2012 and April 2018. First, patients with a prior pacemaker or implantable cardioverter defibrillator (37 patients) were excluded. Second, patients with an aborted procedure and those requiring a cardiopulmonary bypass or surgical conversion (16 patients) were excluded. Finally, patients with missing information for clinical variables (14 patients) were excluded. The final sample size was 382 .

Institutional review board approval was obtained from the office of human research at the University of Illinois at Peoria, IL. Considering the retrospective nature of this study, the consent waiver was approved. All patients undergoing TAVR were deemed as intermediate or high risk for SAVR by the local cardiothoracic surgery team based on the Society of Thoracic Surgeons (STS) score.

Clinical, electrocardiographic, and echocardiographic data were extracted retrospectively, and every patient had a baseline electrocardiogram (EKG) and echocardiogram done before TAVR. The clinical variables studied included age, gender, body mass index (BMI), STS score, history of hypertension, diabetes, prior myocardial infarction, heart failure with different New York Heart Association functional classes (NYHA Class), atrial fibrillation or flutter, smoking, chronic lung disease, and renal disease requiring dialysis.

Echocardiographic variables included left ventricular internal diameter measured at systole and diastole (LVIDs/LVIDd) and ventricular septal wall thickness.

\section{Outcome comparison}

The primary outcomes included PPM implantation after TAVR. In addition, we looked at hours of stay in the ICU, hospital length of stay (LOS) in days, and readmission at 30 days after TAVR.

Secondary outcomes comprised cost differences between the two groups. We calculated the direct, indirect, and total cost of TAVR admission.

\section{Cost categories}

The direct cost was the cost incurred by individual patient care; it varied with patient volume. It was a combination of "direct fixed" and "direct variable cost." Direct fixed costs did not change with patient volume. It included fixed labor, e.g. salaries and wages; fixed benefits of staff, e.g. health insurance, Federal Insurance Contributions Act (FICA), and 401k; and fixed purchase services, e.g. maintenance contracts, pharmaceuticals, equipment maintenance, and offset expenses. Direct variable costs were a combination of labor and benefits and implants, e.g. TAVR valves, pharmacy, blood supply, lab supplies, repair, and maintenance, and would be higher for complex patients and those with a longer stay.

Indirect costs covered the overhead cost allocated to each case; these costs are not volume-sensitive and cannot be impacted at the bedside, e.g. facility costs, housekeeping, maintenance, and information technology. Total cost was a combination of both direct and indirect costs.

\section{Statistical analyses}

Patients were divided into groups based on PPM status. Baseline characteristics and clinical data were compared among groups. Continuous data were represented as mean \pm SD and categorical data as proportions. The t-test was used to compare continuous variables and Chi-square tests for categorical 


\section{Cureus}

variables.

Adjusted statistical analyses were conducted for clinical and economic outcomes. For ICU hours and length of stay, a generalized linear model with log link and Poisson distribution was used. For the cost outcome (direct cost, indirect cost, and total cost), the generalized linear model with log link and gamma distribution was used. All cost variables were inflated to the 2018 US dollar using the Inpatient Hospital Service Consumer Price Index (CPI).

The key covariate is a PPM status variable. Common covariates for adjusted analysis included age, gender (male), smoking status, Society of Thoracic Surgeons (STS) score, body mass index (BMI), left ventricular internal diameter systolic (LVIDs), left ventricular internal diameter diastolic (LVIDd), septal wall thickness, valve type, valve size, prior NYHA functional class, chronic lung disease, diabetes, dialysis, prior myocardial infarction (MI), prior two-week diagnosis of heart failure, hypertension, atrial fibrillation/flutter, and conduction defect. For logistic analysis of the PPM outcome, the following variables were omitted due to collinearity: valve size, access type, and dialysis.

All calculations were performed using STATA 12 (STATA Corp, Texas, US) and a p-value of less than 0.05 was considered statistically significant.

\section{Results}

\section{Baseline characteristics}

A total of 382 patients were included in this study (Table 1). Of these patients, PPM was seen in 19 (4.97\%) patients. When comparing the two groups, there was no significant difference between age (PPM: $81.6 \mathrm{vs.}$ non-PPM: 80.5; $\mathrm{p}=0.564$ ) and STS score (PPM: 7.5 vs. non-PPM: 6.8; $\mathrm{p}=0.588$ ). Gender was also not statistically different between the two groups (males: PPM: $57.9 \%$ vs. non-PPM: $51.2 \%$; $\mathrm{p}=0.572$ ).

\begin{tabular}{|c|c|c|c|c|}
\hline Variables & All sample $(\mathrm{N}=382)$ & PPM (N=19) & No PPM (N=363) & \\
\hline Continuous variables & Mean (SD) & Mean (SD) & Mean (SD) & P value* \\
\hline Age & $80.5(8.5)$ & $81.6(8.7)$ & $80.5(8.5)$ & 0.564 \\
\hline STS score & $6.9(5.1)$ & $7.5(6.4)$ & $6.8(5.1)$ & 0.588 \\
\hline LVIDs & $3.2(0.8)$ & $3.0(0.9)$ & $3.2(0.8)$ & 0.367 \\
\hline LVIDd & $4.6(0.7)$ & $4.7(0.9)$ & $4.6(0.7)$ & 0.606 \\
\hline Categorical variables & \# of sample (proportion) & \# of sample (proportion) & \# of sample (proportion) & $P$ value* \\
\hline Male & 197 (51.6\%) & $11(57.9 \%)$ & 186 (51.2\%) & 0.572 \\
\hline Smoker & $21(5.5 \%)$ & 2 (10.5\%) & 19 (5.2\%) & 0.324 \\
\hline Valve type & & & & 0.172 \\
\hline Sapien & 44 (11.5\%) & $1(5.3 \%)$ & $43(11.8 \%)$ & \\
\hline Sapien XT & $69(18.1 \%)$ & $1(5.3 \%)$ & $68(18.7 \%)$ & \\
\hline Sapien 3 & 269 (70.4\%) & $17(89.4 \%)$ & $252(69.4 \%)$ & \\
\hline Valve size & & & & 0.071 \\
\hline $20 \mathrm{~mm}$ & $14(3.6 \%)$ & $0(0.0 \%)$ & $14(3.8 \%)$ & \\
\hline 23 mm & 141 (36.9\%) & $5(26.3 \%)$ & $136(37.5 \%)$ & \\
\hline $26 \mathrm{~mm}$ & 153 (40.1\%) & $6(31.6 \%)$ & $147(40.5 \%)$ & \\
\hline $29 \mathrm{~mm}$ & 74 (19.4\%) & 8 (42.1\%) & $66(18.2 \%)$ & \\
\hline Body Mass Index & & & & 0.083 \\
\hline Underweight (<25) & $95(24.9 \%)$ & $3(15.8 \%)$ & $92(25.3 \%)$ & \\
\hline Normal (25 <30) & 133 (34.8\%) & $3(15.8 \%)$ & 130 (35.8\%) & \\
\hline Overweight (30 <35) & $81(21.2 \%)$ & 7 (36.8\%) & $74(20.4 \%)$ & \\
\hline Obesity (>=35) & $73(19.1 \%)$ & $6(31.6 \%)$ & $67(18.5 \%)$ & \\
\hline
\end{tabular}




\section{Cureus}

Septal wall thickness

$<1.1$

$>=1.1$

Access type

Femoral

Trans Aortic

Trans Apical

Trans lliac

Subclavian

Prior-NYHA 2 category

I-II

III-IV

Chronic lung disease

None

Mild

Moderate

Severe

Diabetes

Dialysis

Prior MI

Prior HF

Hypertension

Atrial Fibrillation/Flutter

Conduction Defect
77 (20.2\%)

305 (79.8\%)

$331(86.6 \%)$

$23(6.0 \%)$

$23(6.0 \%)$

$3(0.8 \%)$

$2(0.5 \%)$$$
57 \text { (14.9\%) }
$$

325 (85.1\%)

$83(21.7 \%)$

57 (14.9\%)

$30(7.8 \%)$

$157(41.1 \%)$

$14(3.7 \%)$

$114(29.8 \%)$

105 (27.5\%)

$353(92.4 \%)$

$140(36.6 \%)$

$198(51.8 \%)$
2 (10.5\%)

17 (89.5\%)

$16(84.2 \%)$

2 (10.5\%)

$1(5.3 \%)$

$0(0.0 \%)$

$0(0.0 \%)$

3 (15.8\%)

$16(84.2 \%)$

10 (52.6\%)

$4(21.1 \%)$

$4(21.1 \%)$

$1(5.2 \%)$

7 (36.8\%)

$0(0.0 \%)$

$6(31.6 \%)$

$4(21.1 \%)$

15 (78.9\%)

$8(42.1 \%)$

$16(84.2 \%)$
0.915

0.871

75 (20.7\%)

288 (79.3\%)

315 (86.8\%)

21 (5.8\%)

22 (6.1\%)

$3(0.8 \%)$

$2(0.5 \%)$

54 (14.9\%)

309 (85.1\%)

$79(21.7 \%)$

53 (14.6\%)

$29(8.0 \%)$

$150(41.3 \%)$

0.699

14 (3.9\%)

0.383

$108(29.7 \%)$

0.865

$101(27.8 \%)$

0.519

338 (93.1\%)

0.023

$132(36.4 \%)$

0.613

$182(50.1 \%)$

\section{TABLE 1: Baseline characteristics}

* Chi-square test for categorical variables and t-test for continuous variable

Standard Deviation (SD) and proportion (\%) are by the columns.

Society of Thoracic Surgeons (STS), left ventricular internal diameter systolic (LVIDs), left ventricular internal diameter systolic (LVIDd), heart failure (HF), myocardial infarction (MI)

\section{Clinical variables}

The non-PPM group saw comparatively more patients with hypertension as compared to the PPM group (PPM: 78.9\% vs. non-PPM: 93.1\%; $\mathrm{p}=0.023$ ). The percentage of patients with conduction defect was significantly higher in PPM patients (PPM: 84.2\% vs. non-PPM: 50.1\%; p=0.004). No other clinical variable was statistically significant.

\section{Regression analysis}

After adjusting for other clinical variables, logistic regression (Table 2) showed that patients with larger LVIDs were less likely to receive PPM than those with smaller LVIDs (OR: 0.20, CI: 0.04 - 0.91, p=0.038). No other variable was statistically significant. 


\section{Cureus}

\begin{tabular}{|c|c|c|c|}
\hline Covariates & Odds Ratio & $P$ value & 95\% Confidence Interval \\
\hline Age & 1.05 & 0.199 & $(0.97,1.15)$ \\
\hline Male & 0.24 & 0.152 & $(0.03,1.68)$ \\
\hline Smoker & 5.14 & 0.148 & $(0.55,47.24)$ \\
\hline STS score & 1.04 & 0.507 & $(0.92,1.17)$ \\
\hline \multicolumn{4}{|l|}{ BMI (Ref: Normal) } \\
\hline Underweight & 0.85 & 0.865 & $(0.13,5.55)$ \\
\hline Overweight & 3.77 & 0.104 & $(0.76,18.65)$ \\
\hline Obese & 5.57 & 0.050 & $(1.00,31.05)$ \\
\hline LVIDs & 0.20 & 0.038 & $(0.04,0.91)$ \\
\hline LVIDd & 4.38 & 0.077 & $(0.85,22.55)$ \\
\hline Septal wall thickness >=1.1 (Ref: <1.1) & 1.21 & 0.831 & $(0.20,7.06)$ \\
\hline \multicolumn{4}{|l|}{ Valve type (Ref: Sapien) } \\
\hline Sapien XT & 1.24 & 0.890 & $(0.05,27.49)$ \\
\hline Sapien 3 & 4.69 & 0.232 & $(0.37,59.21)$ \\
\hline \multicolumn{4}{|l|}{ Valve size (Ref: 23mm) } \\
\hline 26 mm & 2.19 & 0.347 & $(0.05,27.49)$ \\
\hline $29 \mathrm{~mm}$ & 7.07 & 0.090 & $(0.37,59.21)$ \\
\hline Prior NYHA III-IV & 0.77 & 0.746 & $(0.16,3.66)$ \\
\hline \multicolumn{4}{|l|}{ Chronic lung disease (Ref: None) } \\
\hline Mild & 0.88 & 0.869 & $(0.19,4.00)$ \\
\hline Moderate & 1.29 & 0.745 & $(0.27,6.20)$ \\
\hline Severe & 0.32 & 0.430 & $(0.01,5.35)$ \\
\hline Diabetes & 0.52 & 0.290 & $(0.15,1.74)$ \\
\hline Prior Ml & 1.18 & 0.787 & $(0.35,3.91)$ \\
\hline Prior HF & 1.01 & 0.986 & $(0.21,4.73)$ \\
\hline Hypertension & 0.25 & 0.091 & $(0.05,1.24)$ \\
\hline Atrial Fibrillation/Flutter & 1.11 & 0.848 & $(0.35,3.57)$ \\
\hline Conduction Defect & 3.72 & 0.057 & $(0.96,14.38)$ \\
\hline Constant & $1.9 \mathrm{E}-06$ & 0.020 & $(7.37 \mathrm{E}-10,0.16)$ \\
\hline
\end{tabular}

TABLE 2: Adjusted logistic regression result (outcome: PPM, $n=368$ )

The following variables were omitted due to collinearity: valve size, access type, and dialysis. Valve size of $20 \mathrm{~mm}$ (14 observations) was omitted due to no observation in the PPM group.

Society of Thoracic Surgeons (STS) score, body mass index (BMI), left ventricular internal diameter systolic (LVIDs), left ventricular internal diameter diastolic (LVIDd), myocardial infarction (MI), heart failure (HF)

\section{Multivariable analysis}

Patients receiving PPM stayed an average of 12 hours more in the ICU than the non-PPM group (48.6 vs. 36.7 hours; $\mathrm{p}<0.001$ ) (Table 3). Length of stay in the hospital, although longer for the PPM group, was not statistically significant (PPM: 4.21 vs. non-PPM: 3.40; $\mathrm{p}=0.116$ ). 


\section{Cureus}

\begin{tabular}{|c|c|c|c|c|}
\hline Outcomes & PPM (N=19) Mean $(95 \% \mathrm{Cl})$ & No PPM (N=363) Mean $(95 \% \mathrm{Cl})$ & Difference Mean (95\% Cl) & P value \\
\hline ICU hours ${ }^{1}$ & $48.6(45.2,52.0)$ & $36.7(36.1,37.3)$ & $11.9(8.4,15.3)$ & $<0.001$ \\
\hline Length of stay ${ }^{1}$ & $4.21(3.23,5.18)$ & $3.40(3.21,3.59)$ & $0.80(-0.19,1.80)$ & 0.116 \\
\hline Total cost ${ }^{2}$ & $\$ 81,701(72162,91240)$ & $\$ 71,487(69660,73315)$ & $\$ 10,213(453,19973)$ & 0.040 \\
\hline Direct $\operatorname{cost}^{2}$ & $\$ 53,862(47862,59862)$ & $\$ 46,774(45636,47912)$ & $\$ 7,087(952,13223)$ & 0.024 \\
\hline Pharmacy & $\$ 1,029(290,1768)$ & $\$ 860(664,1056)$ & $\$ 168(-554,891)$ & 0.648 \\
\hline Blood & $\$ 52(-42,148)$ & $\$ 827(-398,2052)$ & $-\$ 774(-2003,454)$ & 0.217 \\
\hline Laboratory & $\$ 601(414,788)$ & $\$ 632(580,685)$ & $-\$ 31(-219,156)$ & 0.742 \\
\hline Room & $\$ 5,568(3326,7810)$ & $\$ 4,001(3609,4393)$ & $\$ 1,566(-684,3817)$ & 0.173 \\
\hline Supply & $\$ 315(-24,654)$ & $\$ 193(120,266)$ & $\$ 121(-198,442)$ & 0.457 \\
\hline Therapy & $\$ 980(531,1429)$ & $\$ 779(682,876)$ & $\$ 201(-245,647)$ & 0.377 \\
\hline Imaging & $\$ 487(284,690)$ & $\$ 430(388,473)$ & $\$ 57(-149,263)$ & 0.588 \\
\hline Miscellaneous cost & $\$ 46,076(42838,49314)$ & $\$ 39,205(38604,39806)$ & $\$ 6,871(3561,10180)$ & $<0.001$ \\
\hline Indirect $\cos ^{2}{ }^{2}$ & $\$ 27,850(24177,31523)$ & $\$ 24,709(23991,25427)$ & $\$ 3,141(-622,6904)$ & 0.102 \\
\hline
\end{tabular}

\section{TABLE 3: Multivariable analysis result $(n=382)$}

All costs were converted to 2018 US dollar using the Inpatient Hospital Service Consumer Price Index (CPI). Covariates included pacemaker, age, male, smoking status, STS score, BMI, LVIDs, LVIDd, septal wall thickness, valve type, valve size, access type, prior NYHA, chronic lung disease, diabetes, dialysis, prior MI, prior two-week HF, hypertension, atrial fibrillation/flutter, and conduction defect.

${ }^{1}$ Generalized linear model (GLM) with log link and Poisson distribution adjusting for the above covariates.

${ }^{2}$ Generalized linear model (GLM) with log link and gamma distribution adjusting for the above covariates.

Permanent pacemaker (PPM), body mass index (BMI), New York Heart Association functional (NYHA), Society of Thoracic Surgeon (STS), heart failure (HF), myocardial infarction (MI)

\section{Cost analysis}

PPM implantation significantly increased the total and direct cost for TAVR admission (Table 3). The average total cost for the PPM group was higher by $\$ 10,213$ ( $\$ 81,701$ vs. $\$ 71,487 ; p=0.040$ ). Direct cost was higher for the PPM group by $\$ 7,087$ ( $\$ 53,862$ vs. $\$ 46,774 ; \mathrm{p}=0.024$ ). Indirect cost, although higher for the PPM group, was not statistically different $(\$ 27,850$ vs. $\$ 24,709 ; \mathrm{p}=0.102)$.

Looking at the breakdown of costs (Table 3), the PPM group incurred a higher cost for almost every subcategory, except for blood products and laboratory use. "Miscellaneous costs" were significantly higher for the PPM group by $\$ 6,871$ ( $\$ 46,076$ vs. $\$ 39,205 ; \mathrm{p}<0.001$ ). These miscellaneous costs (Table 4 ) comprised services received by patients during admission that were specific to the patient's co-morbidities and usually not directly related to the TAVR procedure. These do include electrophysiology services, which were clearly involved with all patients in the PPM group. 


\section{Cureus}

\section{Miscellaneous Cost}

Surgery - General (Major)

Electrophysiology

Gastrointestinal Services

Pulmonary Intervention Lab

Psychiatric Emergency Care

Recovery Room Services

IV Nutritional Support

Emergency Services

ER Physicians

Electroencephalography

Hematology services

Urology Clinic

Pulmonary Function Services

PICC Line Team

\section{TABLE 4: Miscellaneous costs}

Peripherally inserted central catheter (PICC)

\section{Readmission and mortality}

PPM implantation increased the odds of readmission rate at 30 days (OR: 1.19, CI: $0.28-4.95, \mathrm{p}=0.802$ ), however, it was not statistically significant (Table 5). We could not calculate 30-day mortality due to the perfect collinearity between PPM and 30-day mortality.

\begin{tabular}{|c|c|c|c|}
\hline Outcomes & Odds Ratio & P value & 95\% Confidence Interval \\
\hline 30-day readmission & 1.19 & 0.802 & $(0.28,4.95)$ \\
\hline 30-day mortality ${ }^{1}$ & NA & & \\
\hline
\end{tabular}

\section{TABLE 5: Adjusted logistic regression result $(n=382)$}

Note: Covariates included pacemaker, age, male, smoking status, Society of Thoracic Surgeons (STS) score, body mass index (BMI), left ventricular internal diameter systolic (LVIDs), left ventricular internal diameter systolic (LVIDd), septal wall thickness, valve type, valve size, prior New York Heart Association (NYHA), chronic lung disease, diabetes, dialysis, prior myocardial infarction (MI), prior two-week heart failure, hypertension, atrial fibrillation/flutter, and conduction defect. Access type was excluded due to multicollinearity.

NA: not available

${ }^{1}$ 30-day mortality was not available for analysis due to perfect collinearity between 30 -day mortality and PPM.

\section{Discussion}

Transcatheter aortic valve replacement has proven itself as a safe alternative to surgical valve replacement in intermediate to high-risk patients with symptomatic aortic stenosis. Post-TAVR conduction abnormalities needing pacemaker placement are well-documented and, furthermore, have been described with greater frequency in TAVR with an incidence ranging from 5\%-22\% [25]. The proliferation of TAVR has increased the need for pacing post-implantation, with literature to date focusing on factors predicting the need for postimplant device therapy, including anatomical factors, type of prosthesis, and underlying conduction abnormalities [26]. Device therapy in this setting has been previously associated with increased length of 
Our facility exclusively utilizes the balloon expandable delivery systems of the Edwards Lifesciences Sapien line of devices, for which prior studies have identified a lower risk of post-implant conduction defects (around 5\%) when compared to self-expanding device delivery systems (around 12\%-39\%) [28]. Our cohort compared well to previously published cohorts, with 19 patients (4.97\%) necessitated pacing therapy [29]. Baseline characteristics between study groups were non-significant based on t-test and chi-squared testing, with the exception of underlying conduction abnormalities. Our study was not adequately powered for identifying independent predictive variables. However, logistic regression analysis of our cohort demonstrated the statistical significance of one observed variable, showing that patients with larger LVIDs were less likely to need PPM implantation. Baseline conduction defects neared statistical significance $(\mathrm{p}=0.057)$. Lastly, there was no significant difference in 30-day readmission rates.

The primary outcomes of our study met statistical significance for increased intensive care stay and total cost in the pacemaker cohort. First, ICU stay, on average, was 11.9 hours longer than that of the nonpacemaker cohort ( $\mathrm{p}=<0.001$, CI 8.4-15.3). Second, post-TAVR PPM implantation was associated with an increased cost of $\$ 10,213$ ( $\mathrm{p}=0.040$ ) when indexed to the 2018 US dollar using the Inpatient Hospital Service Consumer Price Index (CPI). These compared well to the previously quoted figures of $\$ 6620-\$ 11,885$ in prior publications $[25,27]$.

Our trial was novel in that it utilized itemized facility costs for the identification of factors leading to increased cost. Our facility divided costs based on direct patient care (including pharmacy, blood, laboratory, room, supplies, therapy, imaging, and miscellaneous costs) and indirect cost. Of these categories, direct patient care and miscellaneous costs met statistical significance ( $\mathrm{p}=0.024$ and $<0.001$, respectively). The direct cost was identified to be $\$ 7,087$ more in the PPM cohort. The miscellaneous cost was $\$ 6,871$ greater in the PPM cohort, indicative of the involvement of the electrophysiology service and ancillary staff required for a second procedure (Table 3). Based on our cost analysis, charges related to facility overhead costs were not statistically significant. Previously, it has been postulated that increased length of stay and increased utilization of finite facility resources lead to significant cost increases [30], however, conversely, our study demonstrated the primary drivers are procedure-related costs of the PPM implantation with a relatively fixed overhead cost.

\section{Strengths and limitations of the study}

Our calculations have used cost, indexed to the 2018 US dollar using the Inpatient Hospital Service CPI, which is inflation-adjusted for the study period. The detailed cost of direct and indirect expenses has not been extensively described in the literature for PPM implantation in the TAVR population.

The limitations of our study relate primarily to its limited applicability to other implantation centers based on regional and facility variation in costs and protocols related to post-TAVR conduction abnormalities. In addition, the retrospective nature of this study has inherent limitations. Our study was not adequately powered for the identification of independent predictive variables. Thirty-day mortality could not be calculated because of collinearity in the data, which is another limitation of this study.

\section{Conclusions}

This study is the first to date that focused on identifying specific cost drivers related to post-transcatheter aortic valve replacement pacemaker implantation. The increased cost in our cohort was primarily attributed to procedural costs directly related to pacemaker implantation. However, facility utilization costs did not demonstrate significance. The goal of this study is to spur further research on cost reduction given the continued proliferation of TAVR.

\section{Additional Information \\ Disclosures}

Human subjects: Consent was obtained by all participants in this study. University of Illinois College of Medicine Peoria IRB 1 issued approval 1197310-3. DATE: April 11, 2019 TO: Mansoor Ahmad, MD FROM: University of Illinois College of Medicine Peoria IRB 1 STUDY TITLE: [1197310-3] Valve size as a predictor of permanent pacemaker implantation in Edwards Sapien-3 TAVR valves; A single center experience. IRB REFERENCE \#: SUBMISSION TYPE: Continuing Review/Progress Report ACTION: APPROVED APPROVAL DATE: April 11, 2019 EXPIRATION DATE: April 10, 2020 REVIEW TYPE: Expedited Review Thank you for your submission of Continuing Review/Progress Report materials for this research study. University of Illinois College of Medicine Peoria IRB 1 has approved your renewal submission for one year of study. This approval is based on an appropriate risk/benefit ratio and a study design wherein the risks have been minimized. All research must be conducted in accordance with this approved submission. This submission has received Expedited Review based on the applicable federal regulation. PLEASE NOTE: Research must be conducted according to the proposal that was approved by the IRB. Any revisions to the previously approved materials must be approved by this office prior to initiation. Please use the appropriate revision forms for this procedure. Based on the risks, this project requires Continuing Review by this office on an annual basis. 
Please use the appropriate renewal forms for this procedure. When your study is complete, please submit a Final Report to IRBNet. If this study is regulated by the HIPAA Privacy Rule, please retain copies of all records pertaining to this study for a minimum of six (6) years from study closure. Otherwise, please retain research records for a minimum of three (3) years from study closure. The University of Illinois College of Medicine Peoria's (UICOMP) Office of Human Research Oversight (OHRO) will no longer accept local or nonlocal adverse events or safety reports for IRB review that do not meet the definition of an unanticipated problem involving risks to subjects or others (UPIRSO). UPIRSOs are any incident, experience, or outcome that meets all of the following criteria: 1 . are not expected (in terms of nature, severity or frequency) given (a) the research procedures that are described in the protocol-related documents (such as the research protocol and informed consent document); and (b) the characteristics of the subject population being studied; 2 . are related or possibly related to participation in the research; and 3. suggest that the research places subjects or others at greater risk of harm (including physical, psychological, economic, or social harm) than was previously known or recognized. Please report all NON-COMPLIANCE issues or COMPLAINTS regarding this study to this office. If you have any questions, please contact Wendy Bucklin at (309) 680-8633 or wbucklin@uic.edu. Please include your study title and reference number in all correspondence with this office. Animal subjects: All authors have confirmed that this study did not involve animal subjects or tissue. Conflicts of interest: In compliance with the ICMJE uniform disclosure form, all authors declare the following: Payment/services info: All authors have declared that no financial support was received from any organization for the submitted work. Financial relationships: Sudhir Mungee MD. declare(s) Consultant from Edwards Lifesciences. Dr. Sudhir Mungee has been working as a consultant and is a member of the speaker bureau at Edwards Lifesciences. Other relationships: All authors have declared that there are no other relationships or activities that could appear to have influenced the submitted work.

\section{Acknowledgements}

We would like to thank Elizabeth Berryman (Department of Cardiology, UICOMP) for her help with streamlining the transcatheter aortic valve replacement (TAVR) registry, which improved the data analysis; Wendy Bucklin and Melinda Ann Reeter (Institutional Review Board, UICOMP) for their help in understanding the IRB application process; and Kimberly Hartwig (Clinical Research Regulatory and Quality Assurance Coordinator, OSF St. Francis Medical Center) for her help with the process of adding volunteers to our study and guidance through the process of ensuring research quality based on our institutional requirements.

\section{References}

1. Schwarz F, Baumann P, Manthey J, et al.: The effect of aortic valve replacement on survival . Circulation. 1982, 66:1105-1110.

2. Murphy ES, Lawson RM, Starr A, Rahimtoola SH: Severe aortic stenosis in patients 60 years of age or older: left ventricular function and 10-year survival after valve replacement. Circulation. 1981, 64:184-188.

3. Lund O: Preoperative risk evaluation and stratification of long-term survival after valve replacement for aortic stenosis. Reasons for earlier operative intervention. Circulation. 1990, 82:124-139.

4. Leon MB, Smith CR, Mack M, et al.: Transcatheter aortic-valve implantation for aortic stenosis in patients who cannot undergo surgery. N Engl J Med. 2010, 363:1597-1607. 10.1056/NEJMoa1008232

5. Smith CR, Leon MB, Mack MJ, et al.: Transcatheter versus surgical aortic-valve replacement in high-risk patients. N Engl J Med. 2011, 364:2187-2198. 10.1056/NEJMoa1103510

6. Iung B, Cachier A, Baron G, et al.: Decision-making in elderly patients with severe aortic stenosis: why are so many denied surgery?. Eur Heart J. 2005, 26:2714-2720. 10.1093/eurheartj/ehi471

7. Deeb GM, Reardon MJ, Chetcuti S, et al.: 3-year outcomes in high-risk patients who underwent surgical or transcatheter aortic valve replacement. J Am Coll Cardiol. 2016, 67:2565-2574. 10.1016/j.jacc.2016.03.506

8. Van Mieghem NM, Head SJ, de Jong W, et al.: Persistent annual permanent pacemaker implantation rate after surgical aortic valve replacement in patients with severe aortic stenosis. Ann Thorac Surg. 2012, 94:1143-1149. 10.1016/j.athoracsur.2012.04.038

9. Sinhal A, Altwegg L, Pasupati S, et al.: Atrioventricular block after transcatheter balloon expandable aortic valve implantation. JACC Cardiovasc Interv. 2008, 1:305-309. 10.1016/j.jcin.2007.12.009

10. Saji M, Murai T, Tobaru T, Tabata M, Takanashi S, Takayama M: Autopsy finding of the Sapien XT valve from a patient who died suddenly after transcatheter aortic valve replacement. Cardiovasc Interv Ther. 2013, 28:267-271. 10.1007/s12928-012-0153-9

11. Munoz-Garcia AJ, Hernandez-Garcia JM, Jimenez-Navarro MF, et al.: Factors predicting and having an impact on the need for a permanent pacemaker after CoreValve prosthesis implantation using the new Accutrak delivery catheter system. JACC Cardiovasc Interv. 2012, 5:533-539. 10.1016/j.jcin.2012.03.011

12. Nazif TM, Dizon JM, Hahn RT, et al.: Predictors and clinical outcomes of permanent pacemaker implantation after transcatheter aortic valve replacement: the PARTNER (Placement of AoRtic TraNscathetER Valves) trial and registry. JACC Cardiovasc Interv. 2015, 8:60-69. 10.1016/j.jcin.2014.07.022

13. Ledwoch J, Franke J, Gerckens U, et al.: Incidence and predictors of permanent pacemaker implantation following transcatheter aortic valve implantation: analysis from the German transcatheter aortic valve interventions registry. Catheter Cardiovasc Interv. 2013, 82:569-577. 10.1002/ccd.24915

14. De Carlo M, Giannini C, Bedogni F, et al.: Safety of a conservative strategy of permanent pacemaker implantation after transcatheter aortic CoreValve implantation. Am Heart J. 2012, 163:492-499. 10.1016/j.ahj.2011.12.009

15. Chorianopoulos E, Krumsdorf U, Pleger ST, Katus HA, Bekeredjian R: Incidence of late occurring bradyarrhythmias after TAVI with the self-expanding CoreValve ${ }^{\circledR}$ aortic bioprosthesis. Clin Res Cardiol. 2012, 101:349-355. 10.1007/s00392-011-0398-9 
16. Bagur R, Rodes-Cabau J, Gurvitch R, et al.: Need for permanent pacemaker as a complication of transcatheter aortic valve implantation and surgical aortic valve replacement in elderly patients with severe aortic stenosis and similar baseline electrocardiographic findings. JACC Cardiovasc Interv. 2012, 5:540-551. 10.1016/j.jcin.2012.03.004

17. Calvi V, Conti S, Pruiti GP, et al.: Incidence rate and predictors of permanent pacemaker implantation after transcatheter aortic valve implantation with self-expanding CoreValve prosthesis. J Interv Card Electrophysiol. 2012, 34:189-195. 10.1007/s10840-011-9634-5

18. van der Boon RM, Nuis RJ, Van Mieghem NM, et al.: New conduction abnormalities after TAVI-frequency and causes. Nat Rev Cardiol. 2012, 9:454-463. 10.1038/nrcardio.2012.58

19. Abdel-Wahab M, Mehilli J, Frerker C, et al.: Comparison of balloon-expandable vs self-expandable valves in patients undergoing transcatheter aortic valve replacement: the CHOICE randomized clinical trial. JAMA. 2014, 311:1503-1514. 10.1001/jama.2014.3316

20. Erkapic D, De Rosa S, Kelava A, Lehmann R, Fichtlscherer S, Hohnloser SH: Risk for permanent pacemaker after transcatheter aortic valve implantation: a comprehensive analysis of the literature. J Cardiovasc Electrophysiol. 2012, 23:391-397. 10.1111/j.1540-8167.2011.02211.x

21. Jilaihawi H, Chakravarty T, Weiss RE, Fontana GP, Forrester J, Makkar RR: Meta-analysis of complications in aortic valve replacement: comparison of Medtronic-Corevalve, Edwards-Sapien and surgical aortic valve replacement in 8,536 patients. Catheter Cardiovasc Interv. 2012, 80:128-138. 10.1002/ccd.23368

22. Rodes-Cabau J: Transcatheter aortic valve implantation: current and future approaches . Nat Rev Cardiol. 2011, 9:15-29. 10.1038/nrcardio.2011.164

23. Aljabbary T, Qiu F, Masih S, et al.: Association of clinical and economic outcomes with permanent pacemaker implantation after transcatheter aortic valve replacement. JAMA Netw Open. 2018, 1:180088. 10.1001/jamanetworkopen.2018.0088

24. Ahmad M, Patel JN, Kim M, et al.: Cost impact of pacemaker implantation after transcatheter aortic valve replacement. Catheter Cardiovasc Interv. 2019, 93:19-22.

25. Al-Ogaili A, Fugar S, Okoh A, et al.: Trends in complete heart block after transcatheter aortic valve replacement: a population based analysis. Catheter Cardiovasc Interv. 2019, 1-8. 10.1002/ccd.28156

26. Doshi R, Decter DH, Meraj P: Incidence of arrhythmias and impact of permanent pacemaker implantation in hospitalizations with transcatheter aortic valve replacement. Clin Cardiol. 2018, 41:640-645. 10.1002/clc. 22943

27. Cahill TJ, Chen M, Hayashida K, et al.: Transcatheter aortic valve implantation: current status and future perspectives. Eur Heart J. 2018, 39:2625-2634. 10.1093/eurheartj/ehy244

28. Husser O, Pellegrini C, Kessler T, et al.: Predictors of permanent pacemaker implantations and new-onset conduction abnormalities with the SAPIEN 3 balloon-expandable transcatheter heart valve. JACC Cardiovasc Interv. 2016, 9:244-254. 10.1016/j.jcin.2015.09.036

29. Bhardwaj A, Ramanan T, Sawant AC, et al.: Quality of life outcomes in transcatheter aortic valve replacement patients requiring pacemaker implantation. J Arrhythm. 2018, 34:441-449. 10.1002/joa3.12065

30. Babaliaros V, Devireddy C, Lerakis S, et al.: Comparison of transfemoral transcatheter aortic valve replacement performed in the catheterization laboratory (minimalist approach) versus hybrid operating room (standard approach): outcomes and cost analysis. JACC Cardiovasc Interv. 2014, 7:898-904. 10.1016/j.jcin.2014.04.005 